\title{
Pell graceful labeling of graphs
}

\author{
D. Muthuramakrishnan ${ }^{1 *}$ and S. Sutha ${ }^{2}$
}

\begin{abstract}
In this paper, we introduce a new concept of Pell graceful labeling as follows. An injective function $f$ from $V(G)$ into $\left\{0,1,2, \ldots, p_{q}\right\}$ is Pell graceful if the induced edge labeling $f^{*}(u v)=|f(u)-f(v)|$ is a bijection onto the set $\left\{p_{1}, p_{2}, \ldots, p_{q}\right\}$. A graph $G(p, q)$ which admits a Pell graceful labeling is called a Pell graceful graph, where $p_{q}$ is the $q^{\text {th }}$ Pell number in the Pell sequence. Here, Pell graceful labeling of some family of graphs are obtained. Its non-existence are established.
\end{abstract}

\section{Keywords}

Pell sequence, Pell graceful labeling, Pell graceful graph.

\section{AMS Subject Classification}

$05 \mathrm{C} 78$

1,2 Department of Mathematics, National College, Trichy-620001, Tamil Nadu, India.

*Corresponding author: ${ }^{1}$ dmuthuramakrishnan@gmail.com; ${ }^{2}$ sutha_muruga@rediffmail.com

Article History: Received 2 April 2019; Accepted 11 July 2019

\section{Contents}

1 Introduction

508

2

References

\section{Introduction}

All graphs considered here are simple, finite and undirected. The terms not defined in this paper are used as in Harary [2]. This paper presents results on graph labeling.

Labeled graphs find their applications in Coding Theory and Communication Network Addressing.

Rosa introduced the concept of graceful labeling $f$ of a $(p, q)$ graph $G$ as follows: $f$ is a graceful labeling if $f$ is an injection from $V(G)$ to the set $\{0,1,2, \ldots, q\}$ such that when each edge $u v$ is assigned the label $|f(u)-f(v)|$, the resulting edge labels are distinct.

By following Acharya and Hegde, a new type of labeling called Fibonacci graceful labeling is introduced. The numbers $F_{1}=1, F_{2}=2, F_{3}=3, F_{4}=5, \ldots$ is the sequence of Fibonacci numbers.

As an extension to Fibonacci graceful labeling, in this paper, we introduce Pell graceful labeling. Here, Pell graceful labeling of certain families of graphs are discussed. Also its non-existence are established.

\section{Main Results}

Definition 2.1. Let $G(p, q)$ be a graph. A injective function $f$ from $V(G)$ into $\left\{0,1,2, \ldots, F_{q}\right\}$, where $F_{q}$ is the $q^{\text {th }}$ Fibonacci number is said to be Fibonacci graceful if the induced edge labeling $f^{*}(u v)=|f(u)-f(v)|$ is a bijection onto the set $\left\{F_{1}, F_{2}, \ldots, F_{q}\right\}$.

If a graph $G(p, q)$ admits a Fibonacci graceful labeling then $G$ is called a Fibonacci graceful graph.

As an extension to Fibonacci graceful labeling, we introduce Pell graceful labeling.

Definition 2.2. Let $G(p, q)$ be a graph. An injective function $f$ from $V(G)$ into $\left\{0,1,2, \ldots, p_{q}\right\}$ where $p_{q}$ is the $q^{\text {th }}$ Pell number in the Pell sequence is said to be Pell graceful if the induced edge labeling $f^{*}(u v)=|f(u)-f(v)|$ is a bijection onto the set $\left\{p_{1}, p_{2}, \ldots, p_{q}\right\}$.

If a graph $G(p, q)$ admits a Pell graceful labeling then $G$ is called a Pell graceful graph.

Remark 2.3. The classic Pell sequence is obtained as follows:

$$
\begin{gathered}
p_{0}=0, \\
p_{1}=1
\end{gathered}
$$

and

$$
p_{n+1}=2 p_{n}+p_{n-1} \text { for all } n \geq 1 \text {. }
$$

(i.e.) $\{0,1,2,5,12,29,70, \ldots\}$ is the Pell sequence. 


\section{Illustration 2.1}

In Fig. 2.1, we provide an example of a Pell graceful labeling of a graph.

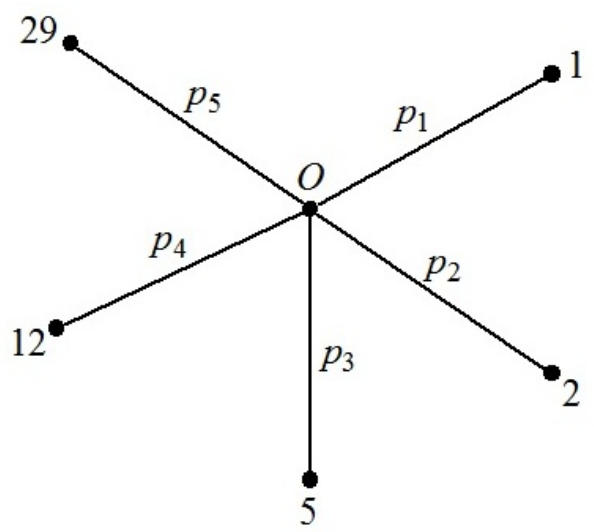

Fig. 2.1

\section{Observation 2.1}

1. The edge number $p_{q}$ has to be induced from the adjacent vertex labels 0 and $p_{q}$. Therefore 0 and $p_{q}$ are ought to be vertex labels in a Pell graceful graph.

2. All vertices adjacent to the vertex labeled with 0 have to receive Pell numbers as their labels.

3. If $\left\{l_{1}(=0), l_{2}, l_{3}, \ldots, l_{n}\left(=p_{q}\right)\right\}$ is a set of vertex labels of a Pell graceful graph then changing each label $l_{i}$ to $p_{q}-l_{i}$ also gives a Pell graceful labeling of the graph.

Theorem 2.4. The cycle $C_{3}$ is not a Pell graceful graph.

Proof. Suppose $C_{3}$ is a Pell graceful graph, then there exists an injective function $f: V(G) \rightarrow\left\{0,1,2, \ldots, p_{q}\right\}$ such that the induced edge labels are Pell numbers $\left\{p_{1}, p_{2}, p_{3}\right\}=\{1,2,5\}$.

Let $u, v, w$ be the vertices of the cycle $C_{3}$. By Observation $2.1(1), f(u)=0, f(v)=p_{3}=5$.

Let $f(w)=x$. Then $f^{*}(u v)=p_{3}$ as in Fig. 2.2.

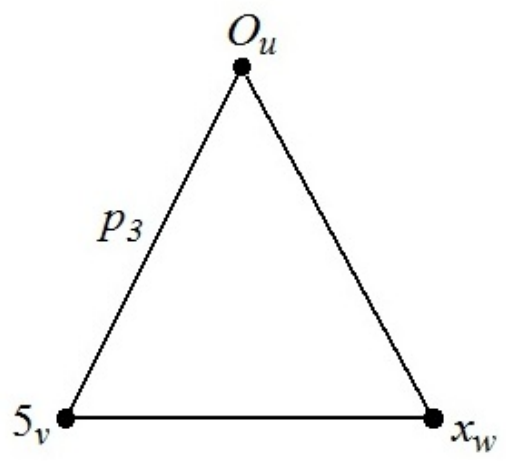

Fig. 2.2
By Observation 2.1(2), $x$ has to receive the Pell number 1 or 2.

If $x=1$, then $f^{*}(v w)=4$ which is not a Pell number, $a \Rightarrow \Leftarrow$.

If $x=2$, then also $f^{*}(v w)=3$ which is not a Pell number, $a \Rightarrow \Leftarrow$ to $f$ is a Pell graceful labeling.

Hence, $C_{3}$ is not a Pell graceful graph.

\section{Observation 2.2}

The absolute difference of any two Pell numbers (except 1 and 2) is not a Pell number.

Theorem 2.5. The wheel $W_{3}$ is not a Pell graceful graph.

Proof. Suppose $W_{3}$ is a Pell graceful graph then there exists an injective function $f: V(G) \rightarrow\left\{0,1,2, \ldots, p_{q}\right\}$ such that the induced edge labels are Pell numbers $\left\{p_{1}, p_{2}, \ldots, p_{6}\right\}=$ $\{1,2,5,12,29,70\}$.

Let $u_{1}, u_{2}, u_{3}, u_{4}$ be the vertices of $W_{3}$ and the ordinary labeling of $W_{3}$ is denoted as in Fig. 2.3.

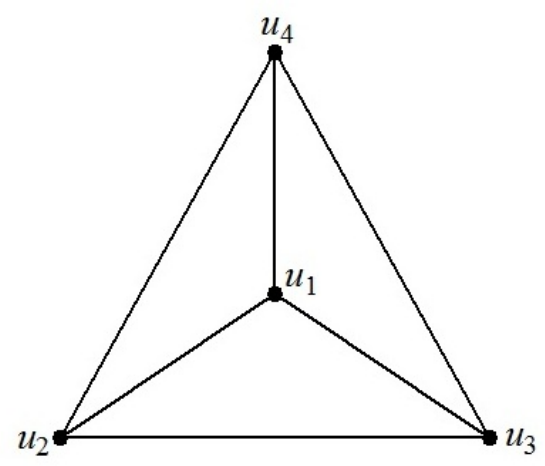

Fig. 2.3

Case 1: $f\left(u_{1}\right)=0$

By Observation 2.2(2), the vertices $u_{2}, u_{3}, u_{4}$ are ought to be labeled with Pell numbers.

By Observation 2.2(1), assume without loss of generality that $f\left(u_{2}\right)=70$.

Then $u_{3}$ and $u_{4}$ cannot hold 1 and 2 as their vertex labels. For, otherwise

$$
f^{*}\left(u_{1} u_{3}\right)=f^{*}\left(u_{3} u_{4}\right)=1, a \Rightarrow \Leftarrow .
$$

Now by Observation 2.2(3), $f$ cannot be a Pell graceful labeling.

Case 2: $f\left(u_{2}\right)=0$ (say)

Then either $f\left(u_{1}\right), f\left(u_{3}\right)$ or $f\left(u_{4}\right)=70$.

Let $f\left(u_{1}\right)=70, f\left(u_{3}\right)=x, f\left(u_{4}\right)=y$ (say).

By Observation 2.2(2), $x$ and $y$ are Pell numbers and cannot be from the set $\{1,2\}$.

Again by Observation 2.2(3), $f$ cannot be a Pell graceful labeling.

Hence, $W_{3}$ is not a Pell graceful graph. 
Theorem 2.6. Every path $P_{n}$ of length $n$ is Pell graceful for all $n \geq 1$.

Proof. Let $P_{n}$ be a path of length $n$.

Let $\left\{v_{0}, v_{1}, \ldots, v_{n}\right\}$ be its vertex set and $\left\{e_{1}, e_{2}, \ldots, e_{n}\right\}$ be its edge set where $e_{i}=v_{i-1} v_{i}$ for $i=1,2, \ldots, n$ as denoted in Fig. 2.4.

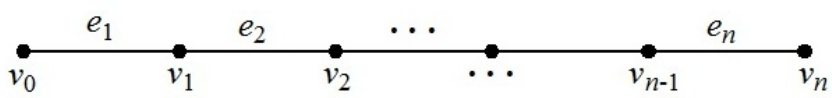

Fig. 2.4: Ordinary labeling of $P_{n}$

We know that

$$
\begin{gathered}
\left|V\left(P_{n}\right)\right|=n+1, \\
\left|E\left(P_{n}\right)\right|=n .
\end{gathered}
$$

First we label the vertices as follows:

Consider the following labeling $f$ on $V\left(P_{n}\right)$.

$$
\begin{gathered}
f\left(v_{0}\right)=0 \\
f\left(v_{i}\right)=p_{n}-p_{n-1}+p_{n-2} \ldots+(-1)^{i-1}+p_{n-(i-1)}
\end{gathered}
$$

for $i=1,2, \ldots, n$.

Here, the induced edge labels are distinct Pell numbers.

Hence, the path $P_{n}$ is Pell graceful for all $n \geq 3$.

\section{Illustration 2.2}

The Pell graceful labeling of $P_{8}$ is given in Fig. 2.5.

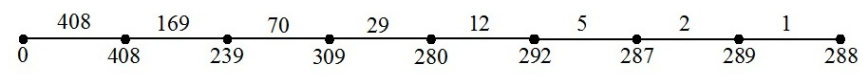

Fig. 2.5: Pell graceful labeling of $P_{8}$

\section{Illustration 2.3}

The Pell graceful labeling of $P_{9}$ is given in Fig. 2.6.

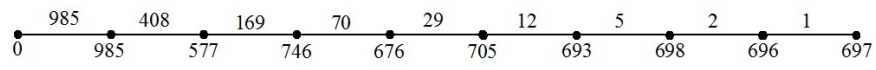

Fig. 2.6: Pell graceful labeling of $P_{9}$

Definition 2.7. An olive tree $O_{n}$ is a collection of $n$ paths joined in one of the end vertices, where the $i^{\text {th }}$ path has length $i$.

Theorem 2.8. Olive trees $O_{n}$ are Pell graceful for all $n \geq 3$.
Proof. Let $O_{n}$ be the olive tree having $n$ paths of length $1,2, \ldots, n$ adjoined at one vertex $v_{0}$.

Let the vertices of $O_{n}$ be $\left\{v_{0}, v_{11}, v_{12}, \ldots, v_{1 n}, v_{21}, v_{22}, \ldots\right.$, $\left.v_{2(n-1)}, v_{31}, v_{32}, \ldots, v_{3(n-2)}, \ldots, v_{n(1)}\right\}$.

The ordinary labeling of $O_{n}$ be as given in Fig. 2.7.

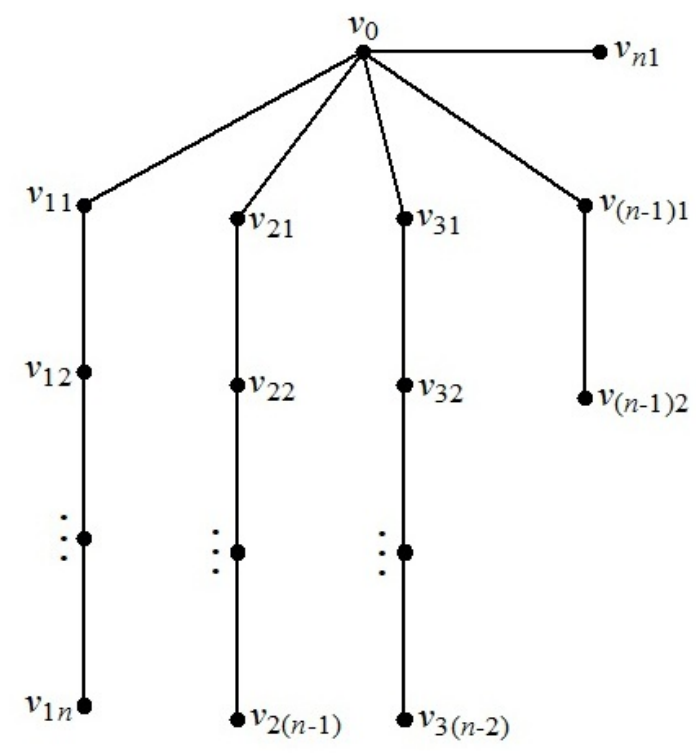

Fig. 2.7: Ordinary labeling of olive trees $O_{n}$

Clearly

$$
\left|V\left(O_{n}\right)\right|=\frac{n^{2}+n+2}{2}
$$

and

$$
\left|E\left(O_{n}\right)\right|=\frac{n(n+1)}{2}=m
$$

Consider the following labeling $f$ for $O_{n}$

$$
\begin{array}{rlrl}
f\left(v_{0}\right) & =0 & \\
f\left(v_{i 1}\right)=p_{m+1-i} & i=1,2, \ldots, n \\
f\left(v_{i 2}\right)=f\left(v_{i 1}\right)-p_{m+1-n-i} & i=1,2, \ldots, n-1 \\
f\left(v_{i 3}\right)=f\left(v_{i 2}\right)-p_{m+2-2 n-i} & i=1,2, \ldots, n-2 \\
f\left(v_{i 4}\right)=f\left(v_{i 3}\right)-p_{m+4-3 n-i} & i=1,2, \ldots, n-3
\end{array}
$$

Continuing in this manner, subtracting the appropriate Pell numbers from $f\left(v_{i 4}\right), f\left(v_{i 5}\right), \ldots$, we get the label for $v_{i 5}, v_{i 6}, \ldots$.

Thus, the induced edge labels are distinct Pell numbers. Hence, the olive trees $O_{n}$ are Pell graceful for all $n \geq 3$.

\section{Illustration 2.4}

Pell graceful labeling of $O_{3}$ is given in Fig. 2.8 


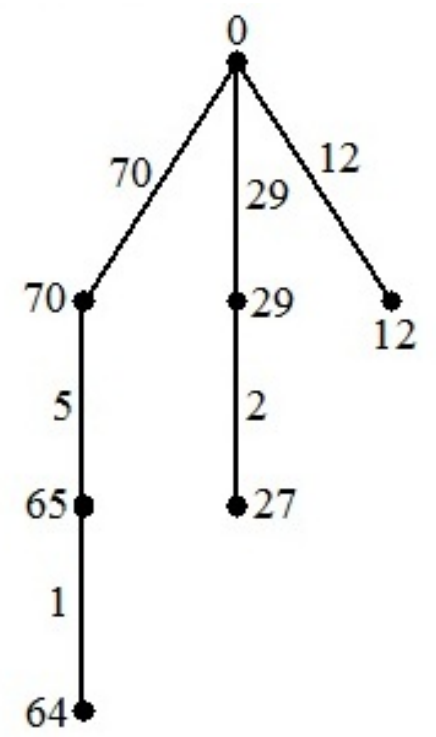

Fig. 2.8: Pell graceful labeling of $\mathrm{O}_{3}$

\section{Illustration 2.5}

Pell graceful labeling of $O_{4}$ is given in Fig. 2.9.

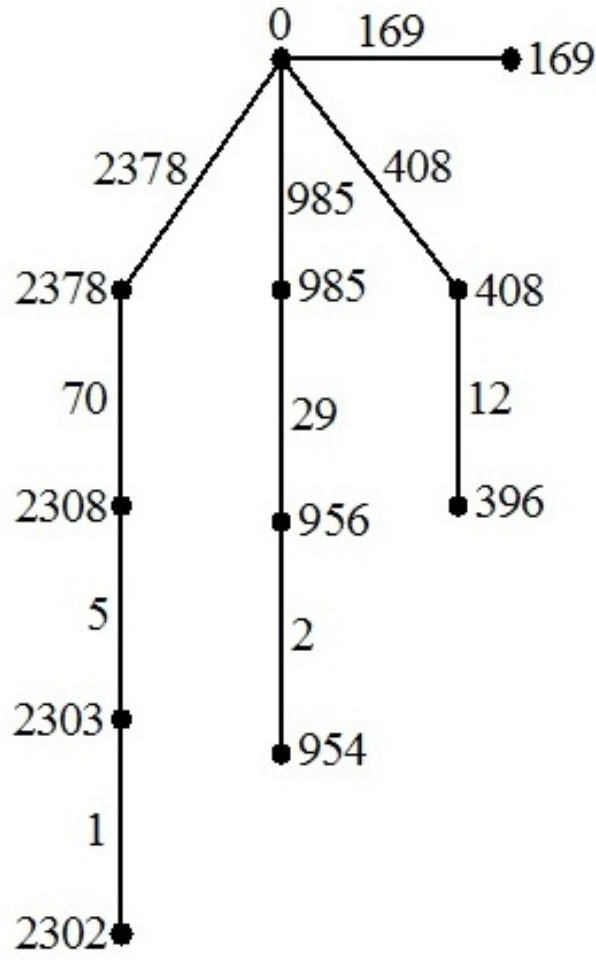

Fig. 2.9: Pell graceful labeling of $O_{4}$

Theorem 2.9. The Combs $P_{n} \odot K_{1}$ are Pell graceful for all $n \geq 3$.

Proof. Let the vertices of $P_{n} \odot K_{1}$ be

$\left\{u_{1}, u_{2}, \ldots, u_{n+1}, v_{1}, v_{2}, \ldots, v_{n+1}\right\}$ and the edges of $P_{n} \odot K_{1}$ be as denoted in Fig. 2.10.

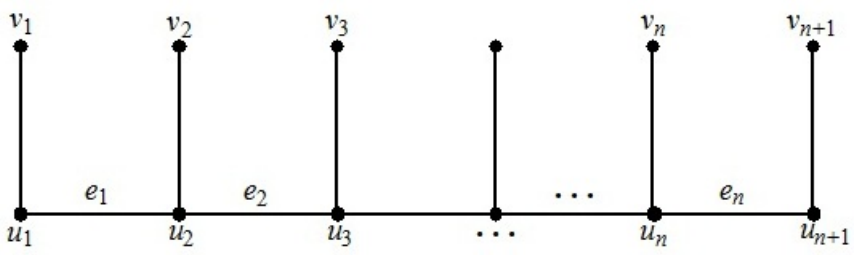

Fig. 2.10: Ordinary labeling of $P_{n} \odot K_{1}$

We know that

$$
\left|V\left(P_{n} \odot K_{1}\right)\right|=2 n+2=p
$$

and

$$
\left|E\left(P_{n} \odot K_{1}\right)\right|=2 n+1=q .
$$

We first label the vertices of $P_{n} \odot K_{1}$ as follows.

Define $f: V\left(P_{n} \odot K_{1}\right) \rightarrow\left\{0,1,2, \ldots, p_{q}\right\}$ by

Case 1: for $n=3$

$$
\begin{aligned}
& f\left(u_{2}\right)=0 \\
& f\left(v_{1}\right)=p_{2}+1 \\
& f\left(v_{2}\right)=p_{4} \\
& f\left(v_{3}\right)=28 \\
& f\left(u_{2 i-1}\right)=p_{2 i-1} \quad \text { for } i=1,2
\end{aligned}
$$

Case 2: for $n \geq 4$

$$
\begin{aligned}
& f\left(u_{n+1}\right)=0 \\
& f\left(u_{n}\right)=p_{2 n+1} \\
& f\left(u_{i}\right)=p_{2 n+1}-\sum_{j=0}^{(n-1)-i} p_{2 n-2 j} \\
& \text { for } i=1,2, \ldots, n-1 \\
& f\left(v_{i}\right)=p_{2 n+1}-p_{2}-\sum_{j=0}^{(n-1)-i} p_{2 n-2 j} \\
& \text { for } i=1 \\
& f\left(v_{i}\right)=p_{2 n+1}-p_{2 i-1}-\sum_{j=0}^{(n-1)-i} p_{2 n-2 j} \\
& \text { for } i=2,3, \ldots, n-1 \\
& f\left(v_{n}\right)=p_{2 n+1}-p_{2 i-1} \\
& f\left(v_{n+1}\right)=1
\end{aligned}
$$

The induced edge labels are distinct Pell numbers. Hence, the Combs $P_{n} \odot K_{1}$ are Pell graceful for all $n \geq 3$.

\section{Illustration 2.6}

Pell graceful labeling of $P_{4} \odot K_{1}$ is given in Fig. 2.11. 


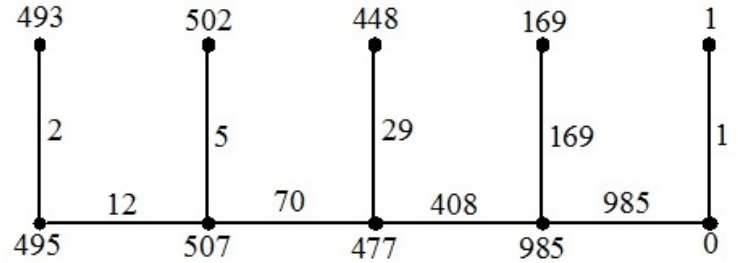

Fig. 2.11: Pell graceful labeling of $P_{4} \odot K_{1}$.

\section{Illustration 2.7}

Pell graceful labeling of $P_{5} \odot K_{1}$ is given in Fig. 2.12.

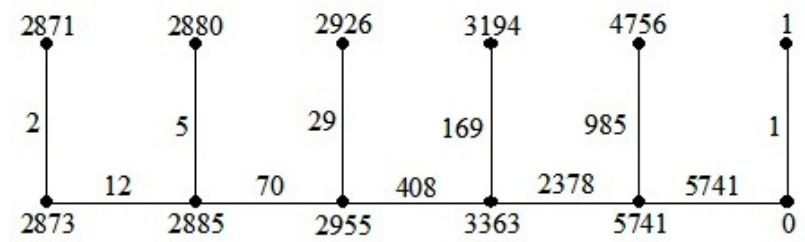

Fig. 2.12: Pell graceful labeling of $P_{5} \odot K_{1}$.

Theorem 2.10. complete graph $K_{n}$ is not a Pell graceful for all $n \geq 3$.

Proof. Let $\left\{v_{1}, v_{2}, \ldots, v_{n}\right\}$ be the vertex set of $K_{n}$. Clearly, each vertex $v_{i}$ is adjacent to every other vertex $v_{j} \forall j$.

Let $v_{1}$ be labelled with 0 . Then, in order to receive $p_{q}$ as edge label, by Observation 2.5(1).

$f\left(v_{j}\right)=p_{q}$ for some $j$ say $j=2$. (i.e.)

$$
\begin{aligned}
& f\left(v_{1}\right)=0, \\
& f\left(v_{2}\right)=p_{q}
\end{aligned}
$$

Now

$$
f^{*}\left(v_{1} v_{2}\right)=p_{q} .
$$

By Observation 2.5(2), every vertex of $K_{n}$ has to be a Pell number.

By Observation 2.7, the difference is not a Pell number, which is a contradiction to the fact that

$$
f^{*}(E(G))=\left\{p_{1}, p_{2}, \ldots, p_{q}\right\} .
$$

Hence, $K_{n}$ is not a Pell graceful graph for all $n$.

\section{References}

[1] J.A. Gallian, A Dynamic Survey of Graph Labeling, The Electronic Journal of Combinatorics, DS6 (2017).

[2] F. Harary, Graph Theory, Addison-Wesley Reading, 1972.

[3] K.M. Kathiresan, S. Amutha, Fibonacci Graceful Graphs, Ars Combin. (To appear).
[4] A. Rosa, On certain valuations of the vertices of a graph, Theory of Graphs (Inter. Symposium, Rome, July 1966), Gordon and Breach, N.Y. and Dunod Paris (1967), 349355 .

[5] G. Sethuraman and P. Selvaraju, Gracefulness of arbitrary super subdivisions of graphs, Indian J. Pure Appl. Math., 32(7) (2001), 1059-1064.

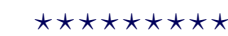

ISSN(P):2319- 3786

Malaya Journal of Matematik $\operatorname{ISSN}(\mathrm{O}): 2321-5666$

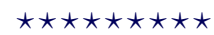

